\title{
ASO Visual Abstract: Stromal Tumor-Associated Macrophage Infiltration Predicts Poor Clinical Outcomes in Muscle-Invasive Bladder Cancer Patients
}

\author{
Shengming Jin, $\mathrm{MD}^{1,2}$, Han Zeng, $\mathrm{MD}^{3}$, Zhaopei Liu, $\mathrm{MD}^{3}$, Kaifeng Jin, $\mathrm{MD}^{3}$, Chunnan Liu, $\mathrm{MD}^{3}$, \\ Sen Yan, $\mathrm{MD}^{3}$, Yanze $\mathrm{Yu}, \mathrm{MD}^{3}$, Runze You, $\mathrm{MD}^{3}$, Hongyi Zhang, $\mathrm{MD}^{3}$, Yuan Chang, $\mathrm{MD}^{1,2}$, \\ Le $\mathrm{Xu}, \mathrm{MD}^{4}$, Jiejie $\mathrm{Xu}, \mathrm{MD}, \mathrm{PhD}^{3}$, Zewei Wang, $\mathrm{MD}^{5}$ (1), and $\mathrm{Yu} \mathrm{Zhu}, \mathrm{MD}^{1,2}$ \\ ${ }^{1}$ Department of Urology, Fudan University Shanghai Cancer Center, Shanghai, China; ${ }^{2}$ Department of Oncology, Shanghai \\ Medical College, Fudan University, Shanghai, China; ${ }^{3}$ Department of Biochemistry and Molecular Biology, School of \\ Basic Medical Sciences, Fudan University, Shanghai, China; ${ }^{4}$ Department of Urology, Ruijin Hospital, Shanghai Jiao Tong \\ University School of Medicine, Shanghai, China; ${ }^{5}$ Department of Urology, Zhongshan Hospital, Fudan University, \\ Shanghai, China
}

Stromal tumor-associated macrophage infiltration could be an independent prognosticator for muscle-invasive bladder cancer (MIBC) patients, and also possesses the potential to guide chemotherapy and immunotherapy decision making in MIBC (https://doi.org/10.1245/s10434021-11155-y).

Supplementary Information The online version contains supplementary material available at https://doi.org/10.1245/s10434021-11262-w.
DISCLOSURE Shengming Jin, Han Zeng, Zhaopei Liu, Kaifeng Jin, Chunnan Liu, Sen Yan, Yanze Yu, Runze You, Hongyi Zhang, Yuan Chang, Le Xu, Jiejie Xu, Zewei Wang, and Yu Zhu declare no potential conflicts of interest.

Publisher's Note Springer Nature remains neutral with regard to jurisdictional claims in published maps and institutional affiliations.

Shengming Jin, Han Zeng, Zhaopei Liu, and Kaifeng Jin contributed equally to this work.

(C) Society of Surgical Oncology 2022

Z. Wang, MD

e-mail: zwwang12@fudan.edu.cn

Y. Zhu, MD

e-mail: yuzhu10@fudan.edu.cn 